\title{
Correlation between morphology and ambipolar transport in organic field-effect transistors
}

\author{
Th. B. Singh, ${ }^{\text {a) }}$ S. Günes, N. Marjanović, and N. S. Sariciftci \\ Linz Institute for Organic Solar Cells (LIOS), Physical Chemistry, Johannes Kepler University Linz, Austria \\ R. Menon \\ Department of Physics, Indian Institute of Science, Bangalore-560012, India
}

\begin{abstract}
Attaining ambipolar charge transport in organic field-effect transistors (OFET) is highly desirable from both fundamental understanding and application points of view. We present the results of an approach to obtain ambipolar OFET with an active layer of organic semiconductor blends using semiconducting polymers in composite with fullerene derivatives. Clear features of forming the superposition of both hole and electron-enhanced channels for an applied gate field are observed. The present studies suggest a strong correlation of thin-film nanomorphology and ambipolar transport in field-effect devices
\end{abstract}

\section{INTRODUCTION}

Recently organic and conjugated semiconducting polymers became attractive for fabricating optoelectronic devices such as active matrix displays, ${ }^{1-3}$ all-polymer integrated circuits, ${ }^{4}$ sensors, ${ }^{5}$ electronic tagging, ${ }^{6,7}$ and ring oscillators. ${ }^{8}$ Some of the main advantages of these materials are low cost, solution processable, and large-area printable production possibilities. Recently, ambipolar organic field-effect transistors (OFET) have been demonstrated to be used in the fabrication of complementary logic circuits. ${ }^{9}$ Demonstrations of ambipolar OFETs have been difficult due to the lack of materials that exhibit both reasonably high electron and hole mobilities. This problem arises mostly due to the strong preference of organic materials either to have high electron mobility or hole mobility and not both at the same time. A very little is known so far about ambipolar charge transport mechanism in organic semiconducting polymers and its dependence on the nanomorphology of thin films; as well as the critical dependence of transport properties on the FET device geometry and interfaces.

Ambipolar charge transport has been demonstrated in OFETs using pentacene, ${ }^{10}$ and oligothiophene derivatized with two [60] fullerenes as active layers. ${ }^{11}$ Ambipolarlike transport has been reported in phthalocyanines. ${ }^{12}$ Bilayer approach has been implemented using $p$-type semiconductor and $n$-type semiconductors to demonstrate ambipolar transport in a standard OFET geometry using inorganic dielectrics. ${ }^{13,14}$ Ambipolar transistor is also demonstrated with coevaporated organic semoconductors ${ }^{15}$ and in blend films of $n$-type and $p$-type semiconductors. ${ }^{16,17}$ So far, most of the OFET device fabrication techniques employed are based on standard inorganic $\mathrm{SiO}_{2}$-based dielectrics. Most recently, polymer-based dielectrics have also been considered as suitable gate insulator materials since polymers are technically relevant insulators in view of an "all-organic" OFET

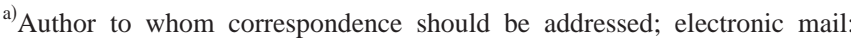
birendra.singh@jku.at
}

structures without the need of any inorganic materials and allowing uniform production technology for all layers in an OFET structure. ${ }^{18,19}$ In this field, we would like to explore a printable ambipolar OFET with a polymeric dielectric as the gate insulator for all solution processed circuits.

We have recently demonstrated a highly photoresponsive OFET (photOFET) using polymer/fullerene blends. ${ }^{20}$ Photoresponsivity as high as $5 \mathrm{~A} / \mathrm{W}$ was obtained from these devices. In this devices, although ambipolar transport is expected from polymer/fullerene blends, features of forming dominantly electron-enhanced channel formation have been observed. Since we fabricate top contact devices, transport properties of the resulting devices strongly depend on the nanomorphology of the thin film. Features of phaseseparated domains are found in these blend thin films. This gives rise to unipolar (dominantly one type of carrier transport) type of transport. In this paper we present the results of an approach to obtain ambipolar OFET with an active layer of three different organic semiconductors in the active blend. Superposition of both hole- and electron-enhanced channels for an applied gate field is clearly observed. A strong correlation of thin-film nanomorphology and ambipolar transport properties is also observed in these devices.

\section{EXPERIMENT}

For the present studies, we have chosen, three different polymer dielectrics, viz., divinyltetramethyldisiloxanebis(benzocyclobutane) (BCB) [Cyclotene ${ }^{\mathrm{TM}}$ ] (Dow Chemicals), polyvinyl alcohol (PVA) [Mowiol ${ }^{\circledR}$ 40-88] (SigmaAldrich), and poly(4-vinyl phenol) (PVP) (Sigma-Aldrich). A scheme of the device geometry is presented in Fig. 1. The device is fabricated on top of the indium tin oxide (ITO)/ glass substrate. $\mathrm{BCB}$ was used as received from Dow Chemicals. In one of the devices, BCB as a dielectric with a 30 wt. \% ratio in mesitylene solution was spin coated at $1500 \mathrm{rpm}$ in $1 \mathrm{~min}$ which resulted with a thickness of $2-\mu \mathrm{m}$-thick film and followed by thermally cross linked at $250{ }^{\circ} \mathrm{C}$ in argon (Ar) atmosphere for $30 \mathrm{~min}$. In some of the 


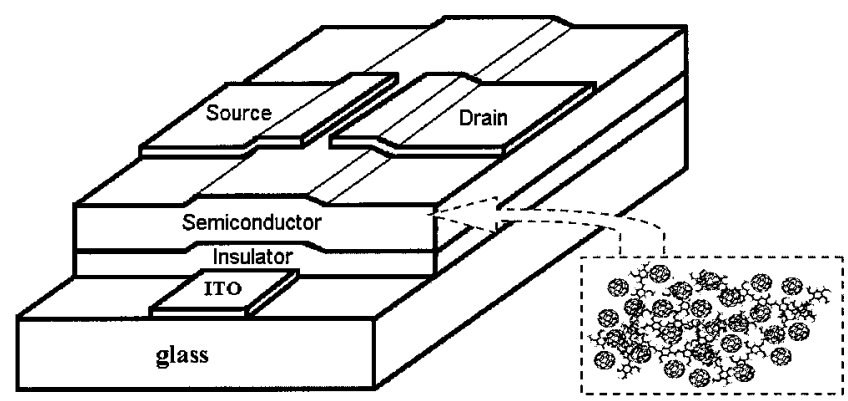

FIG. 1. Schematic of the top contact ambipolar OFET with a channel length of $25 \mu \mathrm{m}$, channel width of $1.5 \mathrm{~mm}$.

devices a polyvinyl alcohol (PVA) (Mowiol@ 40-88) with an average molecular weight of 127000 (Sigma-Aldrich) was spin coated as a gate dielectric from $\mathrm{H}_{2} \mathrm{O}$ solutions. With a $10 \mathrm{wt}$ \% ratio of highly viscous dielectric solution, it gives a thickness of $3.8-\mu \mathrm{m}$ film thickness by spin coating at $1500 \mathrm{rpm}$ in $1 \mathrm{~min}$. In some other devices a poly(4-vinyl phenol) (PVP) with an average molecular weight of 70000 was spin coated as a gate dielectric from $10 \%$ 2- propanol solutions. With a $10 \mathrm{wt}$. \% ratio solution, a thickness of $0.6-1.0-\mu \mathrm{m}$ film by spin coating at $1500 \mathrm{rpm}$ in $1 \mathrm{~min}$ was obtained. Molecular structures of the active semiconductors used are shown in Fig. 2. For the active layer, a blend solution of three organic semiconductors: (poly[2-methoxy-5-(3, 7-dimethyloctyloxy)]-1,4-phenylenevinylene) (MDMOPPV), poly(9,9-dioctyl-fluorenyl-2,7-diyl) end capped with N,N-bis(4-methylphenyl)-4-aniline (PF), and methanofullerene [6,6]-phenyl $\mathrm{C}_{61}$-butyric acid methyl ester (PCBM) (1:1:2 wt. ratio) in chlorobenzene (0.5 wt. \% ratio) were prepared. A blend film of three semiconductors with thickness of $100-120 \mathrm{~nm}$ was obtained by spin coating at $1500 \mathrm{rpm}$ for $1 \mathrm{~min}$ from chlorobenzene solution filtered with a $0.2-\mu \mathrm{m}$ filter in argon atmosphere inside the glovebox. For comparison we have chosen two types of metals, $\mathrm{Au}$ and $\mathrm{LiF} / \mathrm{Al}$ as a drain-source electrode. The top drainsource electrodes, $\mathrm{Au}(60 \mathrm{~nm})$ and $\mathrm{LiF} / \mathrm{Al}(0.6 / 60 \mathrm{~nm})$,
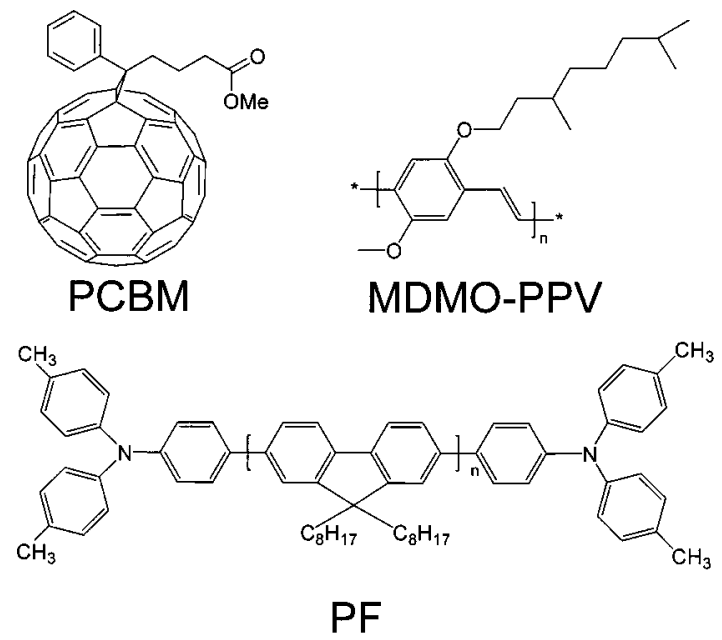

FIG. 2. Chemical structure of semiconductors used in fabricating the ambipolar OFET. PCBM: methanofullerene [6,6]-phenyl $\mathrm{C}_{61}$-butyric acid methyl ester; MDMO-PPV: poly[2-methoxy-5-(3,7-dimethyloctyloxy)]-1,4phenylenevinylene; PF: poly-(9,9-dioctylfluorenyl-2,7-diyl) end capped with $\mathrm{N}, \mathrm{N}$-Bis(4-methylphenyl)-4-aniline. were evaporated under vacuum $\left(3 \times 10^{-6}\right.$ mbar $)$ through a shadow mask. The surface morphology and thickness of the dielectric and blend films were determined with a tapping mode Digital Instrument 3100 atomic force microscope (AFM) and a Dektak-Stylus surface profilometer, respectively. For dielectric constant and capacitance-voltage measurement, separate devices with metal-insulatorsemiconductors (MIS) structures were fabricated in the identical conditions and measured.

\section{RESULT}

Figures 3(a)-3(c) show the height images obtained by AFM in the tapping mode for PVA, BCB, and PVP film, respectively. All three spin cast PVA, BCB, and PVP films have smooth surfaces with a height scale of $5 \mathrm{~nm}$. Morphology studies on MDMO-PPV:PCBM blend film in all the three dielectric surfaces give a film with phase separated domains ca. $200 \mathrm{~nm}$ resulting in cluster formation. ${ }^{20}$ The film morphology of the three semiconductor blend film is shown in Fig. 4. The blend film on PVA has larger phase separated domains as compared to the films on BCB and PVP. Upon adding PF into the MDMO-PPV:PCBM blend, no significant phase separation is observed in the films on BCB and PVP dielectrics as compared to that on PVA, as shown in Figs. 4(a)-4(c). It is presumed that this reduction in phase separation is related to wetting/dewetting of hydrophobic surfaces in both BCB and PVP films. ${ }^{21}$ Factors related to the stresses arising from the superposition of three different molecular structures within the blend may also play an important role.

The complete electrical characteristics for positive and negative drain-source and gate voltages of the devices fabricated from the three semiconductors blend with PVA as a dielectric are shown in Fig. 5. The device features an unipolar transistor with only electron-enhanced channel formation upon positive gate bias $V_{\mathrm{gg}}$. Transport here is assumed to be dominantly due to electrons on the fullerene phase only. The electron mobility $\mu$ is found to be $5 \times 10^{-4} \mathrm{~cm}^{2} / \mathrm{V} \mathrm{s}$. Our previous studies have shown that pristine PCBM mobility is reduced when blended with polymers. ${ }^{20}$ This decrease in carrier mobility can be attributed due to increased disorder upon blending the three components, decreasing the degree of crystallinity compared to the pristine PCBM. Recently ambipolar transport have been reported from annealed PCBM film with a specific OFET geometry with a ring-type drainsource contact and using $\mathrm{SiO}_{2}$ as a dielectric. ${ }^{9,22}$ Our efforts to demonstrate ambipolar transport from pristine PCBM with the geometry used here (Fig. 1) and using a polymeric dielectric have been unsuccessful. The reason for the observation of unipolar transport in the blend film is not known at the moment. Upon changing the PVA dielectric to $\mathrm{BCB}$ dielectric, the very same three semiconductor blend film is seen with different transport properties and ambipolar transport occurred.

Figure 6(a) shows an ambipolar OFET with clear hole enhancement mode as well as electron enhancement mode superimposing closely for an applied $V_{\mathrm{gs}}$. The $p$-channel enhancement mode and $n$-channel enhancement mode occur above $V_{\mathrm{gs}}=-30$ and $30 \mathrm{~V}$, respectively. Below these $V_{\mathrm{gs}}$, the 


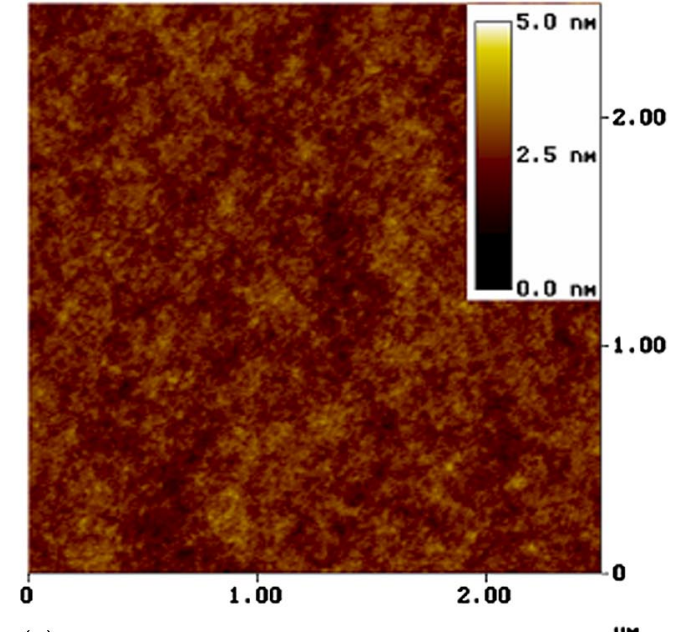

(a)

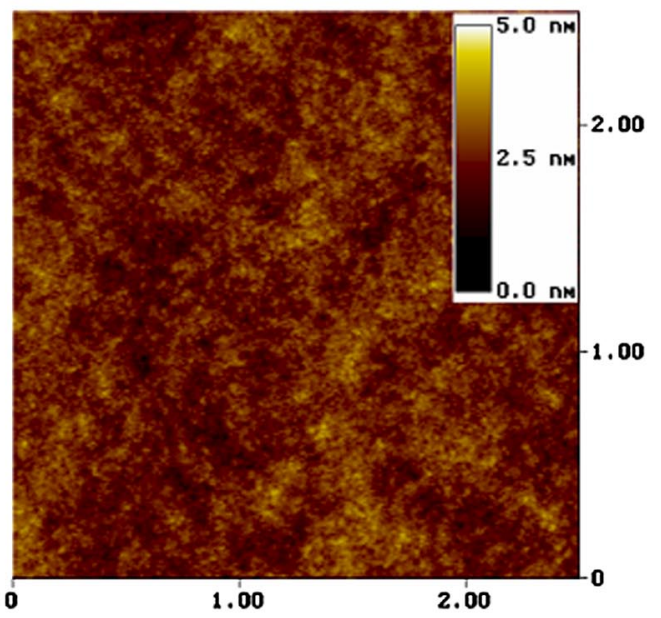

(b)

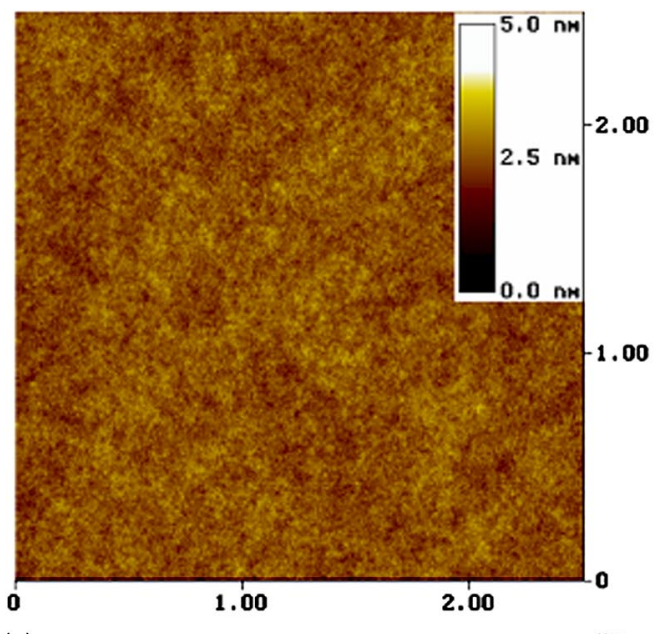

(c)

ym

FIG. 3. (Color online) Tapping mode AFM picture of (a) PVA, (b) BCB, and (c) PVP. All the films have smooth surfaces with a height scale of $5 \mathrm{~nm}$.

drain-source current $I_{\mathrm{ds}}$ due to opposite carrier injection at the drain electrode becomes dominant, as shown in Figs. 6(a) and 6(b). The threshold voltage for formation of enhanced channel is clearly seen in the transfer characteristics, as shown in Fig. 6(b). In Fig. 6(b), when operated in the hole enhancement mode, there exists a deflection point at around $-30 \mathrm{~V}$ where there is a crossover from the electron enhance-

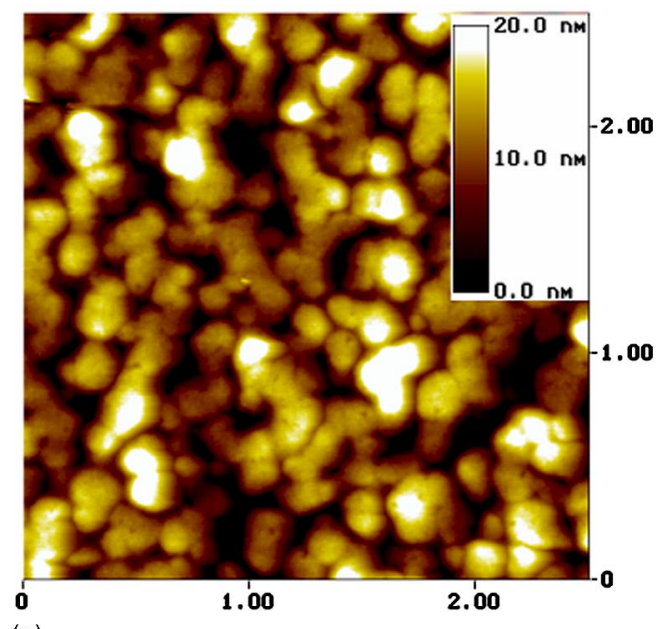

(a)

נM

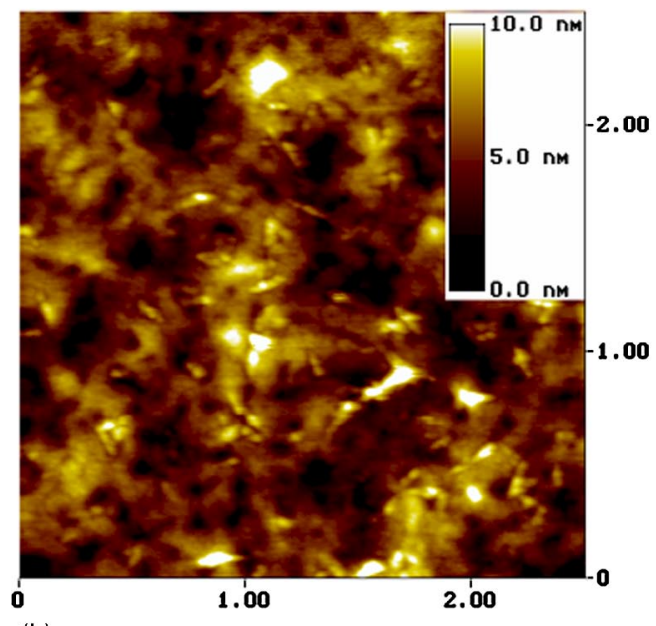

(b)

עM

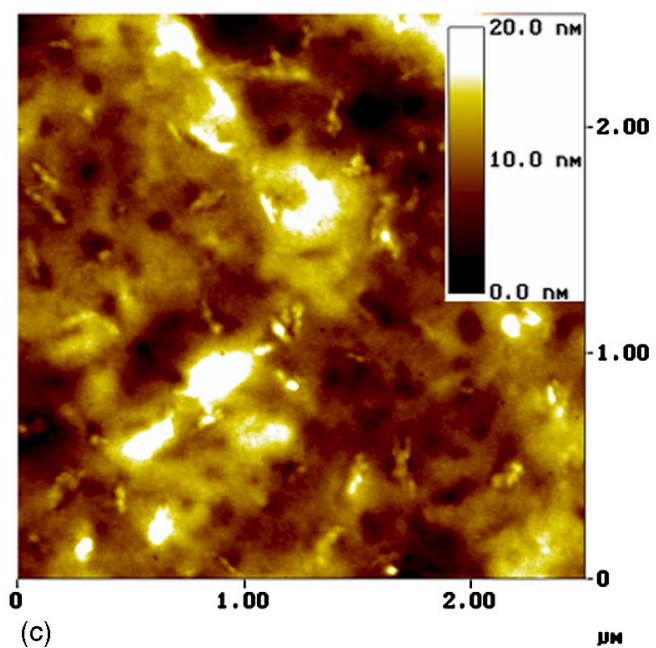

FIG. 4. (Color online) The tapping mode AFM picture of MDMOPPV:PF:PCBM (1:1:2) blend on (a) PVA, (b) BCB, and (c) PVP which indicates large phase separated film on PVA (ca. $200 \mathrm{~nm}$ ) and less phase separated film on BCB and PVP.

ment to the hole enhancement mode. Similarly when operated in the electron-enhanced mode, similar effect occurs with superposition of forming both hole- and electronenhanced channels taking a crossover at deflection point at around $+30 \mathrm{~V}$. The observation of crossover from a hole to an electron-enhanced channel and/or vice versa is a demon- 


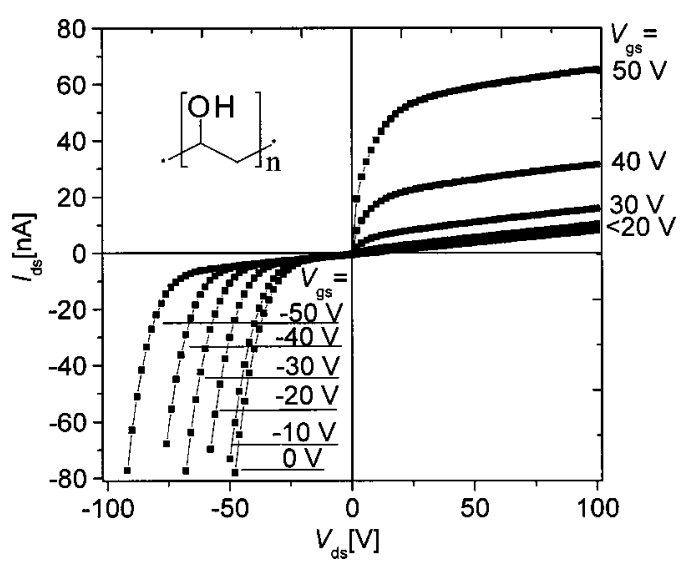

FIG. 5. Typical transistor characteristics of unipolar OFET with MDMOPPV:PF:PCBM (1:1:2) blend film coated on PVA dielectric. Inset: chemical structure of PVA.

stration of an ambipolar OFET. ${ }^{13-17}$ On-off ratio is calculated as ratio of $I_{\mathrm{ds}}$ at $V_{\mathrm{gs}}=100 \mathrm{~V}$ and $I_{\mathrm{ds}}$ at $V_{\mathrm{gs}}=V_{t}$, threshold voltage. Although obtained on-off ratio is 4 and 7 , respectively, for hole- and electron-enhanced modes, for an applied $V_{\mathrm{ds}}, I_{\mathrm{ds}}$ increases quadratically at large $V_{\mathrm{gs}}$. At very large $V_{\mathrm{gs}}$, one type of carrier dominates the charge transport. Crossover
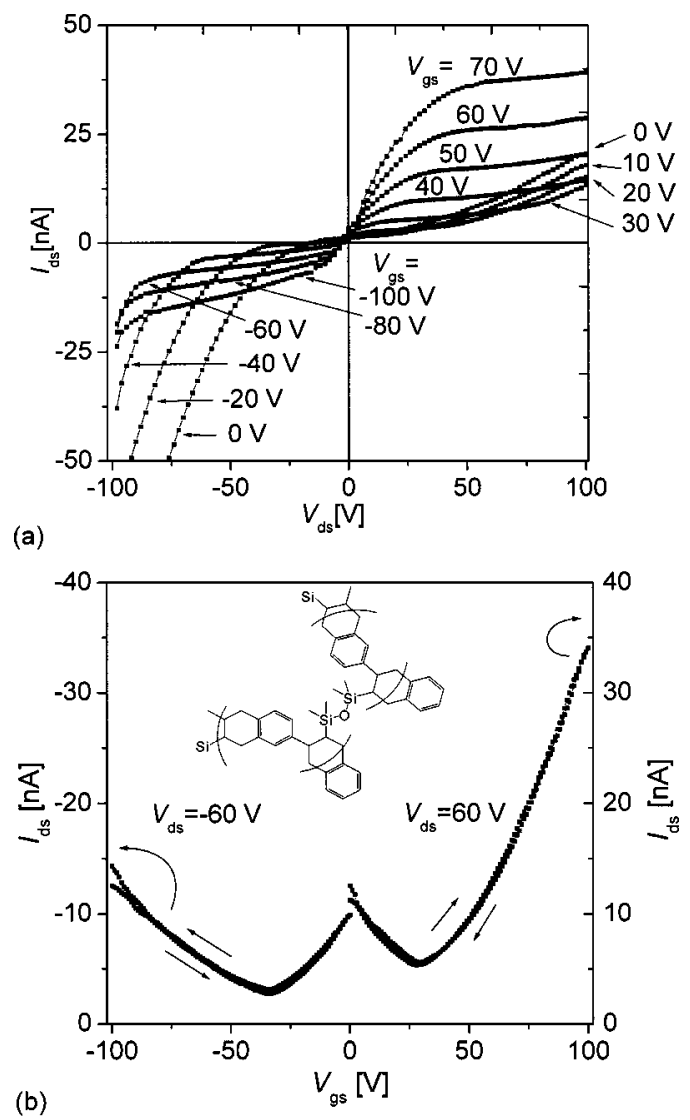

FIG. 6. (a) Typical transistor characteristics of ambipolar OFET with MDMO-PPV:PF:PCBM (1:1:2) blend film coated on BCB dielectric with hole current with pinchoff occurring at $V_{\mathrm{gs}}$ above $-30 \mathrm{~V}$ and electron current with a well-defined pinchoff at $V_{\mathrm{gs}}$ above $+30 \mathrm{~V}$. (b) Transfer characteristics of the ambipolar OFET with hole enhancement mode and electron enhancement mode demonstrating the clear superposition of both hole- and electron-enhanced channel formation for an applied $V_{\mathrm{gs}}$. The arrows indicate the direction sweeping of $V_{\mathrm{gs}}$. Inset: chemical structure of BCB.

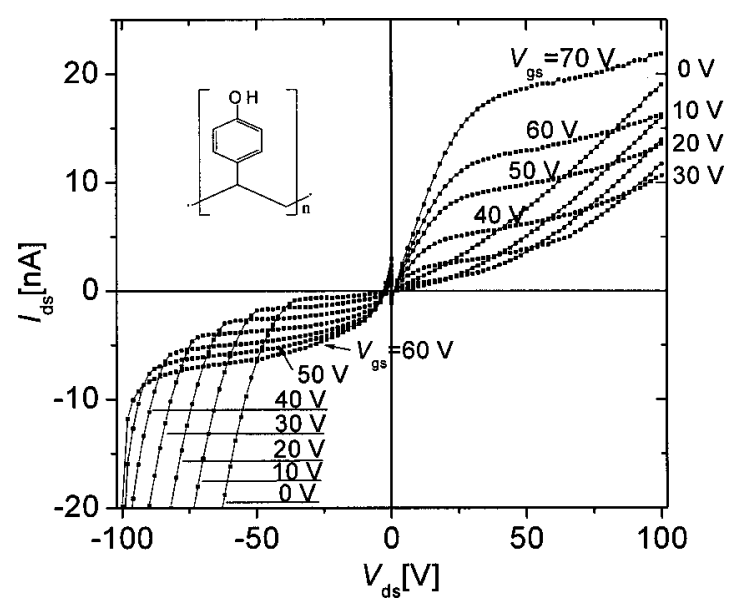

FIG. 7. Typical transistor characteristics of ambipolar OFET with MDMOPPV:PF:PCBM (1:1:2) blend film coated on PVP dielectric demonstrating both hole enhancement mode and electron enhancement mode. Inset: chemical structure of PVP.

from one type of channel to another channel as observed in the present studies are as expected for an ambipolar transistor which attributes to the formation of a quasi $p-n$ junction. ${ }^{23}$ One of the advantages of the present ambipolar OFET is the absence of hysteresis effect in the transfer characteristics unlike the one reported for the polymer/fullerene blend. ${ }^{16}$ On the other hand, charge-carrier mobility is affected by the amorphouslike thin film on the BCB substrate. The charge-carrier mobilities $(\mu)$ are $5 \times 10^{-5}$ and 1 $\times 10^{-5} \mathrm{~cm}^{2} / \mathrm{V}$ s for electron and hole, respectively. Replacing the $\mathrm{Au}$ contacts by $\mathrm{LiF} / \mathrm{Al}$ brings a significant increase in electron mobility up to $10^{-4} \mathrm{~cm}^{2} / \mathrm{V} \mathrm{s}$. This can be attributed to the lowering of barrier for electron injection at $\mathrm{LiF} / \mathrm{Al}$ and PCBM contacts. ${ }^{24,25}$

We will now consider the transfer characteristics of the three semiconductor blend film on top of the PVP dielectric, as shown in Fig. 7. Figure 7 shows an ambipolar transistor with a clear indication of superposition of both electron- and hole-enhanced channels for an applied $V_{\mathrm{gg}}$. From these studies, it is concluded that a blend films on the BCB and PVP films without large phase-separated domains provides ambipolar transport whereas films on PVA provide a phaseseparated domain structure ca. $200 \mathrm{~nm}$ with nanocrystallinelike film which gives rise to unipolar transport properties. In one of the efforts to modify the device structure, OFET with bottom contact was fabricated by evaporating the drainsource electrode in between the dielectric and semiconductor instead of top contact geometry. In such devices, rather poor transport properties are observed presumably due to the low wetting properties of the blend solution on the metal surface. Possible route to reduce the surface free energy of the hydrophilic/hydrophobic surface of the dielectric is by treatment of dielectric surface with vapor-primed n-octadecyltrichlorosilane (OTS) or hexamethyldisilane (HMDS).

\section{CONCLUSION}

An approach to obtain ambipolar OFET with an active layer of three component organic semiconductor blend is 
presented. Clear features in forming the superposition of both hole- and electron-enhanced channels for an applied field are observed. The present studies suggest a strong correlation of thin-film nanomorphology and ambipolar transport in field-effect devices. As such the device represents the basic building block for a solution-processed circuits. Further improvement of the device performance such as on-off ratio is feasible with choosing polymers with higher mobilities and low intrinsic carrier concentrations. One of the suitable solution processable ambipolar materials would be double-cable polymers with regards to both electronic and morphological properties. ${ }^{26}$

\section{ACKNOWLEDGMENTS}

The authors gratefully acknowledge fruitful discussion with Gilles Horowitz from Interfaces, Traitements, Organisation et DYnamique des Systèmes (ITODYS) CNRS, France. Special thanks to $S$. Bauer and R. Schwödiauer for Capacitance-Voltage measurement and fruitful discussion. This work was performed within the Christian Doppler Society's dedicated laboratory on Plastic Solar Cells funded by the Christian Doppler Society and Konarka Austria GesmbH. SG acknowledges the Council of Higher Education (YöK) for the national award scholarship of Turkey.

${ }^{1}$ J. A. Rogers et al., Proc. Natl. Acad. Sci. U.S.A. 98, 4835 (2001).

${ }^{2}$ H. E. A. Huitema et al., Nature (London) 414, 599 (2001).

${ }^{3}$ C. D. Sheraw et al., Appl. Phys. Lett. 80, 1088 (2002).

${ }^{4}$ A. R. Brown, C. P. Jarret, D. M. de Leeuw, and M. Matters, Synth. Met. 88, 37 (1997).

${ }^{5}$ B. K. Crone, A. Dodabalapur, R. Sarpeshkar, A. Gelperin, H. E. Katz, and Z. Bao, J. Appl. Phys. 91, 10140 (2001).

${ }^{6}$ D. M. De Leew, G. H. Gelinck, T. C. T. Geuns, E. Van Veenendaal, E. Cantatore, and B. H. Huisman, Tech. Dig. - Int. Electron Devices Meet.
293 (2002).

${ }^{7}$ P. F. Baude, D. A. Ender, M. A. Haase, T. W. Kelley, D. V. Muyres, and S. D. Theiss, Appl. Phys. Lett. 82, 3964 (2003).

${ }^{8}$ H. Klauk, M. Halik, U. Zschieschang, F. Eder, G. Schmid, and Ch. Dehm, Appl. Phys. Lett. 82, 4175 (2003).

${ }^{9}$ T. D. Anthopoulos, D. M. de Leeuw, E. Cantatore, S. Setayesh, E. J. Meijer, C. Tanase, J. C. Hummelen, and P. W. M. Blom, Appl. Phys. Lett. 85, 4205 (2004).

${ }^{10}$ T. Yasuda, T. Goto, K. Fujita, and T. Tsutsui, Appl. Phys. Lett. 85, 2098 (2004).

${ }^{11}$ Y. Kunugi, K. Takimiya, N. Negishi, T. Otsuo, and Y. Aso, J. Mater. Chem. 14, 2840 (2004).

${ }^{12}$ J. Locklin, K. Shinbo, K. Onishi, F. Kaneko, Z. Bao, and R. C. Advincula, Chem. Mater. 15, 1404 (2003).

${ }^{13}$ A. Dodabalapur, H. E. Katz, L. Torsi, and R. C. Haddon, Appl. Phys. Lett. 68, 1108 (1996).

${ }^{14}$ C. Rost, D. J. Gundalach, S. Karg, and W. Rieß, J. Appl. Phys. 95, 5782 (2004).

${ }^{15}$ K. Tada, H. Harada, and K. Yoshino, Jpn. J. Appl. Phys., Part 2 35, L944 (1996).

${ }^{16}$ E. J. Meijer et al., Nat. Mater. 2, 678 (2003).

${ }^{17}$ A. Babel, J. D. Wind, and S. A. Jenekhe, Adv. Funct. Mater. 14, 891 (2004).

${ }^{18}$ H. Klauk, M. Halik, U. Zschieschang, G. Schmid, W. Radlik, and W. Weber, J. Appl. Phys. 92, 5259 (2002).

${ }^{19}$ H. E. Katz, Ch. Kloc, V. Sunder, J. Zaumseil, A. L. Briseno, and Z. Bao, J. Mater. Res. 19, 1995 (2004).

${ }^{20}$ N. Marjanović, Th. B. Singh, S. Günes, N. S. Sariciftci, R. Schwödiauer, and S. Bauer (unpublished).

${ }^{21}$ B. Viallet, E. Daran, and L. Malaquin, J. Vac. Sci. Technol. A 21, 766 (2003); R. S. Faibish, W. Yoshida, and Y. Cohen, J. Colloid Interface Sci. 256, 341 (2002).

${ }^{22}$ T. D. Anthopoulos, C. Tanase, S. Setayesh, E. J. Meijer, J. C. Hummelen, P. W. M. Blom, and D. M. de Leeuw, Adv. Mater. (Weinheim, Ger.) (Weinheim, Ger.) (in press) (private communication).

${ }^{23}$ G. W. Neudeck, H. F. Bare, and K. Y. Chung, IEEE Trans. Electron Devices ED-34, 344 (1987).

${ }^{24}$ V. D. Mihailetchi et al., Adv. Funct. Mater. 13, 43 (2003).

${ }^{25}$ G. J. Matt, N. S. Sariciftci, and T. Fromherz, Appl. Phys. Lett. 84, 1570 (2004).

${ }^{26}$ A. Cravino and N. S. Sariciftci, Nat. Mater. 2, 360 (2003). 